\title{
Sleep and its importance in adolescence and in common adolescent somatic and psychiatric conditions
}

This article was published in the following Dove Press journal:

International Journal of General Medicine

5 June 2011

Number of times this article has been viewed

\section{Serge Brand' \\ Roumen Kirov ${ }^{2}$ \\ 'Depression and Sleep Research Unit, Psychiatric Hospital of the University of Basel, Basel, Switzerland; ${ }^{2}$ Institute of Neurobiology, Bulgarian Academy of Sciences, Sofia, Bulgaria}

The authors contributed equally to this work
Correspondence: Serge Brand

Depression and Sleep Research Unit, Psychiatric Hospital of the University of Basel, Wilhelm Klein-Strasse 27, 4012 Basel, Switzerland

Tel +4l 6I32 55II4

$\mathrm{Fax}+4 \mid 613255513$

Email serge.brand@upkbs.ch

\begin{abstract}
Restoring sleep is strongly associated with a better physical, cognitive, and psychological well-being. By contrast, poor or disordered sleep is related to impairment of cognitive and psychological functioning and worsened physical health. These associations are well documented not only in adults but also in children and adolescents. Importantly, adolescence is hallmarked by dramatic maturational changes in sleep and its neurobiological regulation, hormonal status, and many psychosocial and physical processes. Thus, the role of sleep in mental and physical health during adolescence and in adolescent patients is complex. However, it has so far received little attention. This review first presents contemporary views about the complex neurobiology of sleep and its functions with important implications for adolescence. Second, existing complex relationships between common adolescent somatic/organic, sleep-related, and psychiatric disorders and certain sleep alterations are discussed. It is concluded that poor or altered sleep in adolescent patients may trigger and maintain many psychiatric and physical disorders or combinations of these conditions, which presumably hinder recovery and may cross into later stages of life. Therefore, timely diagnosis and management of sleep problems appear critical for growth and development in adolescent patients.
\end{abstract}

Keywords: cognitive, psychological, neurobiology, growth, development, sleep physiology, rapid eye movement, non-REM sleep, behavioral disorders, adolescents

\section{Introduction}

Sleep is a universal biological feature in all species, and represents a global state of immobility with greatly reduced responsiveness to environmental stimuli, which can be distinguished from coma or anesthesia by its rapid reversibility. ${ }^{1-3}$ It is by no means a dormant state. When prevented from sleeping, the body tries to recover the lost sleep time. Thus, the existence of sleep rebound after deprivation reveals that sleep is not simply a period of reduced activity or alertness regulated by circadian or ultradian rhythms. ${ }^{4}$ Furthermore, sleep in most vertebrates and all mammal species display a specific architecture and dramatic changes in brain electrophysiology, neurochemistry, and functional anatomy biologically distinguish the different sleep stages from one another. ${ }^{5}$

Although the precise functions of sleep are still beyond a comprehensive understanding, a large body of data show its critical role in many important somatic, cognitive, and psychological processes. Its vital importance is well documented by the fact that sleep deprivation in rodents and flies can cause death more quickly than food deprivation. ${ }^{6}$ It is believed that sleep is beneficial for energy conservation and neuronal recuperation, ${ }^{3}$ synaptic homeostasis, ${ }^{7}$ and brain plasticity. ${ }^{8,9}$ For example, sleep is 
shown to serve many metabolic, immune, thermoregulatory, cardiovascular, and respiratory functions, all responsible for the normal brain and body homeostasis. ${ }^{2,3,7,10}$, Along with these functions, sleep is shown to play a key role in important cognitive and psychological processes, including learning and offline memory consolidation, ${ }^{8,11}$ human heuristic creativity and insightfulness, ${ }^{12-15}$ cognitive abilities, ${ }^{16-21}$ consolidation of emotional memory, ${ }^{22-24}$ and emotional processing ${ }^{25-28}$ including stress-related coping strategies. ${ }^{29}$ Also, a recent study points to a role for sleep in sustaining the so-called default mode network of the brain during quiet wake, which is important for adequate cognitive processes. ${ }^{30}$ Collectively, these data emphasize the beneficial effects of restoring sleep on physical, cognitive, and psychological well-being. ${ }^{1}$ Therefore, sleep is a vital feature, and its situational or pathological alterations can induce maladaptive functioning and a number of psychiatric and physical diseases.

Important maturational sleep alterations in association with changes in many other functions are normally observed during development, with the adolescence being considered an especially vulnerable period during which many physiological, cognitive, and psychological processes sharply mature and are at risk for morbidity. For these reasons, adolescence is drawing increasing attention from broad scientific fields of research. However, the functional importance of sleep in a variety of common conditions during adolescence has so far received less attention.

Adolescence is defined as the period of gradual transition between childhood and adulthood, which are overlapping yet conceptually distinct from the physical changes marking puberty and physical maturation. ${ }^{31,32}$ It is characterized by drastic changes in hormonal and somatic status ${ }^{33,34}$ accompanied by substantial nonlinear changes in brain structural and functional organization and behavior. ${ }^{31,35}$ For example, during this developmental window many psychological functions such as social brain, ${ }^{36,37}$ emotional processing, ${ }^{34,38}$ and executive control ${ }^{39}$ undergo dramatic and rapid changes. The corresponding behavioral signatures that typically signify adolescence include suboptimal decisions and actions; incentive and seeking behaviors; emotional liability; stress in relation to social, working, and academic demands; and increased incidence of unintentional injuries, homicide and suicide attempts, and substance abuse. ${ }^{32,40}$ These behavioral changes are related to the onset of many psychiatric disorders, which increase sharply from childhood to adolescence, ${ }^{41}$ with the lifetime risk for the emergence of mental illness peaking at age $14 .{ }^{42,43}$ Thus, adolescence is the period with nonlinearly increased onset of psychiatric disorders, such as anxiety, posttraumatic stress disorder (PTSD), bipolar disorder (BP), depression, schizophrenia, obsessive-compulsive disorder (OCD), and anorexia nervosa (AN), relative to childhood and adulthood. ${ }^{44-52}$ Also, some of the most common child psychiatric disorders, attention-deficit/hyperactivity disorder (ADHD) and tic disorder (TD), can frequently cross into adolescent depression and OCD or exacerbate in adolescent patients. $^{53-56}$

Importantly, the somatic, neuronal, behavioral, and psychological signatures of adolescence are associated with prominent maturational changes in sleep-wake cycle, and sleep timing, duration, and architecture - parameters during this developmental period reach those commonly observed in adults. ${ }^{57-61}$ These sleep changes are suggested to reflect neurobiological sleep regulatory mechanisms that undergo maturation during adolescence, ${ }^{60-62}$ global synaptic reorganization hallmarking this period, ${ }^{63-66}$ or rapid adaptation of the sleep-wake schedule in response to changes in environmental requirements. ${ }^{57,59}$

Given the vital importance of sleep, it can be concluded that adolescence is a critical period for normal growth and development in which sleep, in a complex association with many other processes, plays an important role. In this review, we will first present contemporary views about the complex neurobiology of sleep and its functions with implications for adolescence. Second, we will discuss existing complex relationships between common adolescent somatic/organic, sleep-related, and psychiatric disorders and certain sleep alterations.

\section{Sleep physiology and architecture}

The heterogeneous nature of sleep can be seen in human and in most animal polysomnographic (PSG) records, which traditionally use electrophysiological techniques including electroencephalography (EEG), electromyography (EMG), and electro-oculography (EOG) to characterize sleep at system levels. Overnight human sleep is characterized by the cyclic occurrence of rapid eye movement (REM) sleep and non-REM sleep, which includes lighter sleep (stages 1 and 2 ) and deeper, slow wave sleep (SWS) (stages 3 and 4). ${ }^{67}$ These distinct sleep stages can be determined by their major electrophysiological signatures. SWS is hallmarked by EEG slow wave activity (SWA) comprising delta (1-4 Hz) waves and slow $\left(<1 \mathrm{~Hz}\right.$ ) oscillations (SO) ${ }^{68,69}$ whereas REM sleep signatures include wake-like EEG activity comprising theta $(\sim 5 \mathrm{~Hz})$, beta $(\sim 15-30 \mathrm{~Hz})$, and gamma $(>30 \mathrm{~Hz})$ rhythms accompanied by swift occurrence of REM upon lack of muscle tone. ${ }^{70-72}$ Stage 2 of non-REM sleep is defined by 
presence of EEG sleep spindles oscillating at 12-15 Hz and K-complexes. ${ }^{67,73}$ Stage 1 of non-REM sleep represents a brief transition from wake to sleep, and is depicted as a replacement of waking EEG by theta or slower EEG rhythms paralleled by the appearance of very slow circular eye movements. ${ }^{74,75}$ Normally, sleep onset begins with a brief period of stage 1 non-REM sleep, which is subsequently followed by sleep deepening marked by the appearance of stage 2 non-REM sleep and a further progressive transition to stages 3 and 4 of SWS. The latter is followed by a relatively short transient of stage 2 non-REM sleep, after which a period of REM sleep appears. This sleep stage's progression, and in particular, the non-REM-REM sleep alternation forms one sleep cycle of approximately 90 minutes duration, and about five or more such sleep cycles are usually observed in normal human overnight sleep. ${ }^{67,74,75}$ Whereas SWS dominates the first half of the night, REM sleep and stage 2 non-REM sleep dominate the second half. This ultradian dynamic reflects the circadian regulation of sleep that is distinguishable from its homeostatic regulation seen after sleep deprivation. ${ }^{76,77}$ A typical PSG graph of a healthy adolescent's overnight sleep is presented in Figure 1A.

\section{Implications for adolescence}

For the purpose of this review, the following implications for adolescence can be ruled out. First, given that sleep displays a specific architecture built up by its distinct stages, it should be considered that the stages' maturation during adolescence undergoes unequivocal changes. For example, a reduction of both SWS and REM sleep EEG signatures at the expense of a relative augmentation of stage 2 non-REM sleep EEG rhythms mark the transition from childhood to adolescence. ${ }^{61}$ This indicates that the distinct sleep stages maturate more or less dissimilarly, and their regulation and functions differ. This issue will be discussed later. Second, other recent studies clearly show that a decline in EEG SWA during SWS occurring from childhood to adolescence topographically follows the global and extensive synaptic reorganization, or "pruning", during adolescence, thus strengthening relevant synaptic networks over irrelevant ones. ${ }^{63-66}$ Therefore, it is the sleep architecture rather than sleep as a whole that matures during adolescence. Sleep architecture is vulnerable to a complex interaction between maturational, situational, and pathological changes in sleep during this period.

\section{Sleep regulation}

The regulation of sleep is active in its own right, and is closely related to its physiology and functions. ${ }^{78}$ The respective neurobiological mechanisms are represented by complex reciprocal interactions between different neuronal populations and their chemical modulators and transmitters in distinct functional states across the sleep-wake cycle..$^{5,79-82}$ Two major brain regions are mostly considered in sleep regulation, especially when the functions of sleep and its alterations in various maturational and pathological conditions are concerned. ${ }^{80,83}$ The first engages neuronal populations located in the diencephalon. Briefly, these include: 1) the suprachiasmatic nucleus of the hypothalamus, which governs the circadian regulation of the sleep-wake cycle through circadian clock genes signaling to the rest peripheral cell oscillators, and couples the functional brain states with the nearby 24-hour light-dark cycle. Its neurons are active during wakefulness and just before sleep onset, thus oppose the homeostatic sleep drive and provide even periods of wake and sleep. 2) The ventrolateral preoptic nucleus of the hypothalamus, which consists of $\gamma$-aminobutyric (GABA)ergic neurons that become prominently active during sleep onset and further in the course of sleep. 3) Hypocretin/Orexin (Hpcr/Orx) neurons in the lateral hypothalamus, which are active during wake and wane during sleep. Notably, along with their wake-promoting role, these neurons also regulate eating, sexual, and locomotor behaviors. ${ }^{83}$ 4) The histamine (His)-containing neurons in the tuberomammillary nucleus of the dorsal hypothalamus, which like Hpcr/Orx neurons are wake-active and wane during sleep. 5) The dorsomedial nucleus of the hypothalamus, which is considered to integrate the hormonal signaling, including hypothalamo-pituitary axis (HPA), with the psychological and physiological functional states across the sleep-wake cycle. ${ }^{84}$

The second brain region engages brainstem or mesopontine and basal forebrain nuclei spread in the reticular ascending system (RAS) and projecting noradrenalin (NA), serotonin (5-hydroxytryptamine, 5-HT), and acetylcholine (Ach) neuromodulatory signals to the upper brain structures including basal ganglia and amygdala, thalamus, hippocampus, and cortex. During wake, the brainstem/mesopontine NA, 5-HT, Ach, and the hypothalamic His neurons projecting to the upper brain structures and cortex are all active, thus sustaining optimal functioning of the different brain states for environmental requirements. ${ }^{5,79,80}$ As sleep deepens from stages 1 and 2 to SWS, all these neurotransmitters progressively decrease their activities, with their lowest during SWS. This results in strongly diminished or lacking ascending projections of RAS neuromodulation to the upper brain structures and cortex, which in turn is responsible for the non-REM sleep EEG signatures originating from 
A

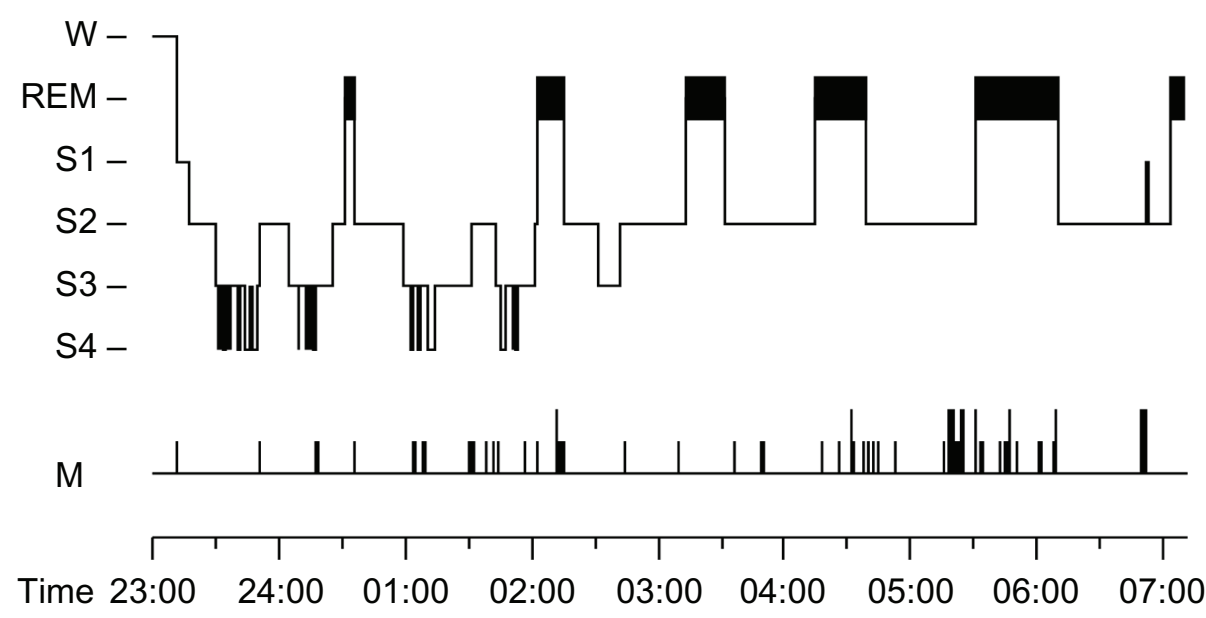

B

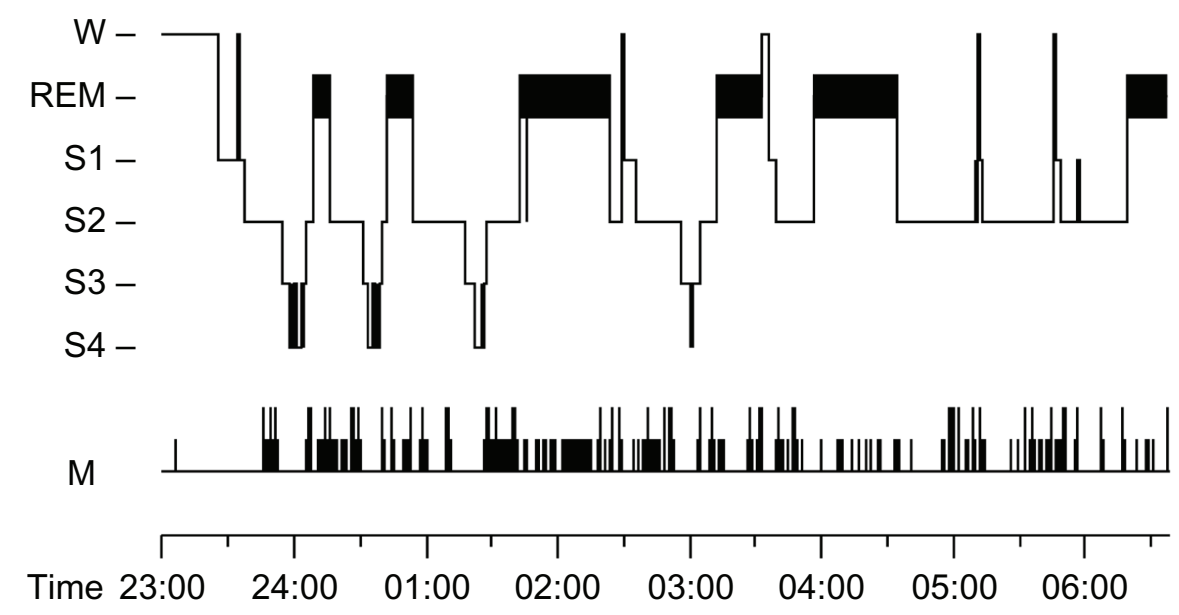

Figure I Representative hypnograms of $\mathbf{A}$ ) healthy adolescent and $\mathbf{B}$ ) adolescent with depression.

Note: Time scale is in hours.

Abbreviations: W, wake; REM, rapid eye movement sleep; SI, stage I of non-REM sleep; S2, stage 2 of non-REM Sleep; S3, stage 3 of non-REM sleep; S4, stage 4 of nonREM sleep; $M$, time of movements.

thalamocortical and corticocortical interplay as reflected by the sleep spindles, K-complexes, and SWA. ${ }^{80,85,86}$

In REM sleep, all NA, 5-HT, and His neurons cease their firing. Instead, an Ach excessive overactivity emerges projecting to the cortex and all subcortical structures and cortical areas, which results in occurrence of the electrophysiological signatures of REM sleep. ${ }^{79,80}$

Further concerning the mesopontine and basal forebrain sleep regulatory systems, it should be noted that they work by reciprocal interactions between the so-called REM-off, silent in REM sleep (NA-adrenergic and 5-HT-ergic neurons in locus coeruleus and dorsal raphe, respectively), and REM-on, active in REM sleep (tegmental and pedunculopontine, Ach-ergic, and glutamatergic neurons) through GABA interneurons and NA-ergic autoreceptors. These mechanisms of sleep regulation are essential for the ultradian alternating expression of non-REM-REM sleep periods. ${ }^{79,80,87,88}$

Concomitantly, important reciprocal interactions among peptides, such as growth hormone (GH)-releasing hormone (GHRH) and corticotropin-releasing hormone (CRH), play a role in ultradian sleep regulation. GHRH is active and GH inhibits HPA hormones, with the CRH as indicated by the cortisol release being suppressed to a minimum during the early, rich in SWS sleep, portion of night. By contrast, HPA activity and cortisol levels reach their peak during late, rich in REM, sleep at night. ${ }^{81,82}$

Although the levels of brain dopamine (DA) activity have not been shown to vary substantially across sleep-wake stages ${ }^{89}$ more recent data indicate an important role for the mesopontine DA-ergic system in the regulation of timing and duration of 
distinct sleep stages, and in particular REM sleep. ${ }^{90,91}$ However, the DA role in sleep regulation is still elusive.

\section{Implications for adolescence}

The following implications for adolescence should be regarded concerning sleep regulation.

Sleep and its regulatory mechanisms maturate from infancy to late adolescence. ${ }^{60}$ For instance, the human newborn exhibits an even distribution of waking, REM sleep, and SWS, spending about 8 hours in each state. After birth, there is a gradual decrease in REM sleep from about 8 hours at birth to about 1 hour by age 15 , beyond which there is a small decrease until senescence. The gain observed in total waking time, from about 8 hours at birth to about 16 hours at maturity, is mostly at the expense of REM sleep duration. ${ }^{60}$ Animal studies indicate that the neural substrates responsible for these developmental changes in sleep-wake cycle are located in the brainstem or mesopontine/basal forebrain nuclei involved in regulation of the ultradian expression of non-REM-REM sleep alternation, as described previously. These sleep regulatory mechanisms have been suggested to comprise the so-called developmental REM sleep-suppressing system, ${ }^{92-94}$ which may be altered and/or not adequately matured in a spectrum of psychiatric disorders during development, including pubertal schizophrenia, panic attacks, PTSD, and anxiety depression - all exhibiting REM sleep overdrive..$^{93,95}$ Moreover, if not properly treated, these disorders can cross into adulthood. Also, the impaired REM sleep regulation during development may lead to occurrence of major depression, insomnia, Alzheimer's, Huntington's, and Parkinson's diseases later in life. ${ }^{93,95}$ Also in this context, depressive disorders are strongly associated with an increased secretion of corticotropin-releasing factor (CRF) and subsequent increased cortisol levels. Both increased CRF and cortisol adversely affect sleep, thus producing delayed sleep onset, shortened REM sleep latency, increased REM sleep, reduced SWS, and increased light sleep. ${ }^{82,95}$ A typical PSG of an adolescent with depression is shown in Figure 1B.

Further, sleep in adolescents displays later sleep onset associated with earlier morning awakenings and relatively shorter duration compared with that in children. This may reflect maturational alterations of sleep's circadian regulation, its adjustment to environmental changes, or both. ${ }^{96,97}$ Moreover, the dysfunctional thoughts, worries, anxiety, ruminations, and unfavorable beliefs adolescents have, all result in hyperarousal, which delays sleep onset, provokes awakenings after sleep onset, and increases early morning awakening. ${ }^{98,99}$ From a neurobiologic and neurocognitive point of view, it should be emphasized that the dorsomedial nucleus of the hypothalamus interacts with both suprachiasmatic and ventrolateralpreoptic nuclei. As described previously, this nucleus is more than a mere relay, and is believed to integrate cognitive and emotional information with neuroendocrinological processes as well as to trigger and modulate physiological arousal. As a result, cognitiveemotional arousal seems to increase physiological arousal and disrupt sleep via the interaction of the hypothalamic nuclei. ${ }^{84}$ Thus, it might be proposed that maturation of circadian sleep regulation or its adjustment to sleep-wake schedule in adolescence are very fine processes that might be substantially affected by the nonlinear behavioral changes during this developmental window.

\section{Contemporary interpretation of the functions of sleep}

Given the heterogeneous nature of sleep, it has been recently well established that its distinct sleep stages and their specific EEG signatures contribute dissimilarly to its functions. In general, whereas non-REM sleep contributes to energy conservation and synaptic homeostasis as reflected by the use-dependent sleep changes, REM sleep is proposed to serve neuronal recuperation and emotional regulation., ${ }^{2,3,7,10,24}$ More specifically, non-REM sleep, particularly SWS, provides conditions for brain plasticity at system and neural levels to take place, whereas REM sleep provides the soundest basis for brain plasticity at synaptic and genetic levels. ${ }^{8,9,100,101}$ Both non-REM sleep and REM sleep provide these conditions through their specific neuromodulatory activities and EEG signatures, as described earlier in the respective sections. Accordingly, the cognitive and psychological functions of non-REM and REM sleep are recently distinctly differentiated.

Recent studies demonstrate that SWS and the portion of non-REM sleep immediately before SWS actively consolidate memories, with the EEG delta waves, SO, and spindles playing a critical role for this memory consolidation. ${ }^{102-104}$ Neuroimaging data strongly indicate that the memory consolidation during SWS involves transfer of previously acquired memories stored in the hippocampus from the hippocampus to the cortex by the so-called hippocampalcortical dialog, ${ }^{105-107}$ which suggests that SWS also provides conditions for a reprocessing or reconsolidation of the previously learned material. ${ }^{108,109}$ Indeed, it is shown that early night sleep that is rich in SWS transforms previously acquired implicit knowledge into explicit knowledge, solving the task. ${ }^{15} \mathrm{~A}$ later study using the same paradigm revealed the 
EEG mechanism of this transformation during SWS: slow spindle (8-12 Hz) EEG activity, which is clearly distinguishable from the use-dependent changes (SWA and fast spindles) in response to presleep task performance. ${ }^{110}$

Another important signature of non-REM sleep stage 2 sleep spindles, is associated with cognitive abilities, ${ }^{20,21,111}$ and a recent study in children demonstrates stage 2 spindles beneficial for coping strategy in response to stress. ${ }^{29}$

REM sleep, its neurobiology and dreaming mental signature have been proposed to serve complex cognitive and psychological functions. ${ }^{78,112,113}$ Indeed, REM sleep has been shown to facilitate access to weak associations, thus promoting human heuristic creativity,,$^{12,13,114,115}$ and late night REM sleep has been demonstrated to preserve previously learned implicit knowledge. ${ }^{15}$ Although it has been consistently found that REM sleep consolidates mostly procedural memory, ${ }^{116-120}$ more recent studies suggest a permissive rather than immediate effect of REM sleep on memory. ${ }^{103,121,122}$ Thus, the role of REM sleep is proposed to be related to integration of recently consolidated memory in a more general and individually specific context. ${ }^{123}$

Notably, REM sleep may have an important role in selective facilitation of affective memories. Earlier studies revealed the social aggression of interaction signature in dreams during REM sleep compared with those during non-REM sleep, ${ }^{26,27}$ which suggests that the process of REM sleep mental activity aids in the resolution of previous emotional conflict. ${ }^{124}$ Indeed, REM sleep and its EEG signature, theta EEG activity, were shown to benefit consolidation of fact-based information with negative emotional salience, thus suggesting that REM sleep has a role in emotional processing. ${ }^{22-24,125}$ However, these features of REM sleep and their respective neural grounds, if improperly set, may lead to the development of a variety of psychiatric disorders with affective component. ${ }^{24,126,127}$ A recent observation in children and adolescents points to a bidirectional role for REM sleep in cognitive abilities and psychopathological expression depending on the presence or absence of psychiatric disorders. $^{18}$

\section{Implications for adolescence}

Considering the above-described sleep stages specific functions, the major implication for adolescence is that preserving normal overnight sleep architecture appears critically important for successful physical, cognitive, and psychological functioning. The sleep architecture of a healthy adolescent in comparison with that of a depressed adolescent is shown in Figure 1. As can be seen in the figure, compared with the PSG of the healthy adolescent (Figure 1A), the PSG of the depressive adolescent is characterized by delayed sleep onset, less time spent in sleep and in SWS, shortened latency to REM sleep, and augmented REM sleep duration, all accompanied by more arousals as reflected in the movement time (Figure 1B). This implies that the depressive adolescent patient experiences far less physical, cognitive, and psychological benefits from his/her sleep compared with the healthy subject.

In this regard, a number of common adolescent conditions beyond highly specific somatic and psychiatric disorders during this developmental period could substantially affect sleep architecture, and thus may lead to maladaptive functioning, including obesity, poor academic achievement, and decremented psychological processing. These conditions include making suboptimal decisions and actions, emotional liability, stress in social relationships with peers, increased incidence of unintentional injuries, violence, substance abuse, and excessive use of online gambling and electronic media. ${ }^{57,59,128-133}$ These behaviors that typically hallmark adolescence ${ }^{32,40}$ are accompanied by psychological and physiological hyperarousal and related chronic sleep loss, ${ }^{98,99}$ which in turn can deteriorate synaptic plasticity, especially during development. ${ }^{134}$ Therefore, such behaviors in adolescence in association with sleep alterations may be regarded as preconditions of developmental psychiatric disorders.

Interestingly, the relationship between these adolescent conditions and altered sleep architecture seem to be bidirectional. For example, poor or insufficient sleep in adolescents is shown to be associated with increased incidents of unintentional injuries and increased risk at suboptimal decisions. ${ }^{135}$ In parallel, excessive use of psychotropic substances, such as alcohol, benzodiazepines, and cannabis, which is associated with suboptimal decisions, in turn produces alterations of sleep architecture including reduction of SWS and REM sleep through action of these substances on GABA-A receptors. ${ }^{82}$ Indeed, such a bidirectional relationship between substance use and sleep disturbances in adolescents is proposed and it is concluded that treatment of both sleep problems and substance use should be considered. ${ }^{136}$

Furthermore, the emotional liability, stress, and homicidal/ suicidal thoughts that are characteristic of adolescence are commonly associated with increased HPA activity and substantial increase in both REM sleep and nightmares, and the later sleep alterations are considered as predictors of PTSD and depression in adolescence. ${ }^{137,138} \mathrm{~A}$ bidirectional relationship between psychological adolescent problems and altered sleep architecture is also supported by some epidemiological 
observations. For example, it is reported that among a large sample of 99,668 adolescents aged 12-18 years, shorter sleep duration was related to worsened psychological status. Two years later, the follow-up evaluation of the same sample revealed that the relation between poor sleep and poor psychological status remained stable over time. ${ }^{139}$

Many strategies are proposed to restore altered sleep architectures related to common but not pathological conditions in adolescence, including behavioral intervention and psychotherapy, yet regular physical exercise appears to be one very promising tool. For example, adolescents who played football three times a week displayed better sleep efficiency, more SWS, and less REM sleep compared with age-matched subjects who did not, with this effect being longlasting. ${ }^{140}$ The possible reason for this finding could be that general use-dependent sleep changes in response to the physical activity, which may be suggested to normalize altered sleep architecture.

\section{Common organic diseases and sleep alterations in adolescence}

In this section, altered sleep in adolescents undergoing cleft lip and/or palate surgery and chemotherapy for oncologic diseases is reviewed.

\section{Cleft lip and/or palate}

Orofacial clefts (OFCs) are the most frequent birth defects worldwide. One in 500-700 newborns suffers from OFC. ${ }^{141,142}$

It is still a matter of debate as to whether a repaired cleft lip and/or palate (CL/P) is related to impaired sleep. Since the occurrence of repaired $\mathrm{CL} / \mathrm{P}$ does not seem to lead to impaired sleep ${ }^{143}$ or to affect psychological functioning in children and adolescents, sleep could be adversely affected if breathing is concomitantly impaired due to the occurrence of CL/P. In this respect, MacLean et $\mathrm{al}^{145,146}$ and Muntz et al ${ }^{147}$ reported poor sleep in children and adolescents with $\mathrm{CL} / \mathrm{P}$ in two specific cases: 1) if CL/P was inadequately or only partially repaired; 2) if $\mathrm{CL} / \mathrm{P}$ was associated with further structural abnormalities of the airway. In these cases, the risk of sleep-disordered breathing was increased. Similarly, Muntz et $\mathrm{al}^{147}$ also reported an increased risk of obstructive sleep apnea (OSA), though with significant improvement after surgical repair.

\section{Sleep in children and adolescents undergoing chemotherapy}

Whereas incidence rates for cancer in children and adolescents are very small, accounting for about 44 cases per
1 million individuals per year, ${ }^{148}$ the subjective burden both for parents and children is tremendous. The reason for assessing sleep in children and adolescents suffering from cancer and undergoing chemotherapy stems from the observation that poor sleep is related to poor psychological functioning. Thus, preventing or reducing poor sleep is considered a possible means of reducing any further psychological burden during chemotherapy. However, data are scarce. Walker et $\mathrm{al}^{149}$ investigated subjective sleep patterns and the sleep hygiene of 51 adolescents (range: 10-19 years) prior to and during chemotherapy. Adolescents during chemotherapy reported significantly poorer sleep quality and sleep hygiene behaviors, when compared with normative data from healthy controls. But when compared with normative data from adolescents suffering from chronic pain, the opposite was true, with significantly better sleep quality and sleep hygiene behaviors in adolescents during chemotherapy. Within-group comparisons further revealed that during chemotherapy the sleep-wake pattern was similar to the weekend sleep-wake pattern but dissimilar to the weekday sleep-wake pattern prior to chemotherapy. This pattern of results suggests that during the chemotherapy of this sample a shift occurred to later bedtimes, longer sleep duration, and prolonged sleep in the morning hours. Whereas in this study the underlying mechanisms remained unclear, possible answers are provided by the study conducted by Zupanec et al. ${ }^{150}$ The authors investigated children and adolescents undergoing maintenance chemotherapy for acute lymphoblastic leukemia (ALL) and observed a variety of different factors causing altered sleep in the majority (87\%) of children. Among these, side effects of medication and changed sleep habits were often reported. By contrast, factors such as sleeping with someone, comforting activities and routines, favorable effects of medications, and food and drink were reported to contribute substantially to favorable sleep. Next, Rosen and Brand $^{151}$ investigated sleep and daytime sleepiness in 53 children suffering from various cancers by using polysomnography, multiple sleep latency test, and actigraphy. The authors observed that children with neoplasms of the central nervous system (CNS) involving the hypothalamus, thalamus, and brainstem had the most frequent and severe sleep problems. Moreover, excessive daytime sleepiness was the most common sleep problem, seen in $60 \%$ of children with cancer and in $80 \%$ of children with CNS neoplasms involving the hypothalamus, thalamus, and brainstem. Moreover, sleep-disordered breathing was present in $40 \%$ of the entire group of children with cancer and in $46 \%$ of children with neoplasms in the hypothalamus, thalamus, and brainstem. 
In children suffering from leukemia, 39\% complained of insomnia. The causes of the sleep problems ranged from neurological injury caused by the neoplasm and the CNSdirected treatments to seizures, adenotonsillar hypertrophy, side effects of medication, obesity, pain, anxiety, and drug abuse. Rosen and Brand ${ }^{151}$ further observed that some of the sleep problems were present before the diagnosis of cancer, though most developed after treatment initiation, and that the treatments were generally successful if sleep problems and their causes were correctly identified and treatments were directed to the specific cause of the problem.

To summarize, alterations in sleep patterns are often observed in children and adolescents suffering from cancer and undergoing chemotherapy. If, on one hand, specificity for the cancer medication side effects and concomitant diseases reduce sleep, on the other hand, factors such as sleeping with someone, comforting activities and routines, favorable effects of medications, and food and drink can be considered as protective factors.

\section{Sleep-related disorders in adolescence}

This section will review some commonly observed sleeprelated disorders in adolescence, including OSA, snoring, periodic leg movements in sleep (PLMS), and restless legs syndrome (RLS).

\section{OSA and snoring in children and adolescents}

Estimates of children and adolescents suffering from OSA range from $1 \%-3 \%,{ }^{152-154}$ while regular snoring is observed in up to $12 \%$ of children. ${ }^{154}$ Snoring and OSAs are considered to be closely related to malformations of the oral cavity, namely to adenotonsillar hypertrophy and to congenital malformations often related to CL/P. ${ }^{144}$

OSA during adolescence represents a serious condition that can substantially impair sleep architecture and a number of physical and cognitive functions. The cognitive consequences include daytime somnolence, learning disabilities, and deficient memory consolidation. ${ }^{155-158}$ The physical consequences are described as the so-called metabolic syndrome, which includes obesity, diabetes, immune deficits, and cardiovascular problems. ${ }^{159,160}$ For example, Tran et al investigated 42 children (mean age: 5.8 years) with OSA confirmed by positive findings on PSG and undergoing tonsillectomy and adenoidectomy and 41 control children (mean age: 7.3 years) with no history of snoring. ${ }^{153}$ All children were assessed twice for emotional, social, and behavioral functioning. Before surgery, children with OSA had significantly poorer scores for internalizing and externalizing problems and somatic complaints compared with healthy controls. After tonsillectomy and adenoidectomy, internalizing and externalizing problems and somatic complaints had significantly decreased in children with OSA and they no longer differed statistically from healthy controls. However, Mitchell reported less optimistic findings from 79 children and adolescents aged 3-14 years with OSA undergoing surgical repair. ${ }^{161}$ Whereas sleep and respiratory values, as assessed by polysomnography, generally improved dramatically after surgery, changes in children's quality of life as assessed by parents' judgments were also significant, though, most importantly, adversely related to surgery outcome.

Since adolescents tend to have poor compliance with the noninvasive positive airway pressure (PAP) therapy for OSA, data about the effect of PAP therapy on physical, cognitive, and psychological functioning in children and adolescents are inconsistent. ${ }^{162}$ Yet, a recent study demonstrated that obese adolescents with OSA who adhered to PAP treatment displayed improvement in attention and academic functioning relative to adolescents with OSA who underwent PAP therapy but did not adhere to it. ${ }^{163}$

To summarize, successful surgical intervention in children and adolescents suffering from OSA brings about dramatic improvements in respiratory and sleep outcome. These improvements may be accompanied by improvements in psychological functioning and behavior, ${ }^{153}$ though not necessarily in a global score of quality of life. ${ }^{161}$ The reason for this heterogeneous pattern of results remains elusive, though differences related to samples (sample size, age, degree of OSA, and concomitant malformation of the oral cavity), assessment tools, and surgery outcome may all contribute to this heterogeneous picture. Similarly, PAP treatment shows positive effects on OSA and related daily behaviors in adolescents but only when adolescents adhere to it. ${ }^{163}$

With respect to snoring, Blunden et al investigated the neuropsychological and psychosocial functioning of 64 children. ${ }^{164}$ Of these, 11 were snorers, 13 reported behavioral sleep problems, and 9 reported both snoring and behavioral sleep problems. The remaining 31 were age and gendermatched controls. All children underwent psychological (Wechsler Abbreviated Scale of Intelligence ${ }^{\circledR}$, Children's Memory Scale $^{\circledR}$, Test of Everyday Attention ${ }^{\circledR}$, and Auditory Continuous Performance Test ${ }^{\circledR}$ ) and psychosocial assessment (Child Behavior Checklist). Results showed a differentiated impact of snoring and behavioral sleep problems in 
that snoring was associated with intelligence and attention deficits whereas behavioral sleep problems were associated with memory and behavioral deficits.

To conclude, numerous studies based on different methodologies, samples, and outcome variables suggest that sleep and psychological functioning are also affected in children and adolescents suffering from impaired respiratory functions.

\section{PLMS and RLS in children and adolescents}

PLMS disorder is unique in that the movements occur during sleep. The condition is remarkably periodic. PLMS may occur with other sleep disorders and is related to, but not synonymous with, RLS. The latter is a sensorimotor disorder characterized by an irresistible urge to move the legs, often accompanied by uncomfortable sensations, which is relieved by movement and worse in the evening or night and at rest. ${ }^{165}$ It is believed that alterations in brain dopaminergic systems and iron deficiency cause their core symptoms. ${ }^{166-171}$ Whereas prevalence rates for adults range from 5\%-15\%, data about the rate of PLMS and RLS in children and adolescents are largely inconsistent because these motor sleep disturbances are shown almost always coexisting with ADHD, TD, anxiety, and depression in children and adolescents. ${ }^{168,170,172,173}$ Furthermore, since alterations in brain DA systems and iron deficiency are also shown to underlie ADHD and TD psychopathology in children, ${ }^{55,167,168,174,175}$ is suggested that RLS, ADHD, and TD form a spectrum of comorbid conditions. ${ }^{170,175}$ Interestingly, however, complaints of poor sleep in relation to PLMS and RLS among large samples of children and adolescents were shown to be more in adolescents than in children. ${ }^{170}$

Given the extremely high rate of PLMS asnd RLS comorbidity in ADHD, TD, and other psychiatric conditions in children and adolescents, there is little information about how these motor sleep disorders, if present alone, affect daily functioning. Yilmaz et al investigated 3304 high school adolescents aged 15-18 years. ${ }^{176}$ Participants completed a series of questionnaires related to sleep, RLS, and psychological functioning. Results showed that RLS was diagnosed in $3.6 \%$ of participants. Occurrence of RLS was associated with nocturnal bed-wetting, sleeping difficulty, daytime sleepiness, and, most importantly, with hyperactivity/ inattention. With respect to PLMS and RLS comorbidity in ADHD, Chervin et al investigated 866 children and adolescents aged 2.0-13.9 years. ${ }^{172}$ First, they found that prevalence of RLS was about $17 \%$ and, second, that RLS and PLMS were associated with an increased risk for suffering from inattention and hyperactivity.

Concerning the pharmacological treatment of PLMS and RLS, psychostimulants such as DA agonists have been proved effective; however, given the high rate of both sleep motor disorders in children and adolescents with ADHD, TD, and other psychiatric disorders, this therapy should be adjusted and linked to the leading condition. ${ }^{170}$ In this regard, one promising treatment strategy may be iron supplementation. ${ }^{168,175}$

In conclusion, PLMS and RLS may affect both sleep and daily functioning and thus may lead to impaired development and poor academic achievement in adolescents. However, when subjectively reported or documented by PSG, PLMS and RLS should be treated with increased attention with respect to concomitant psychiatric disorders.

\section{Sleep and common adolescent psychiatric disorders}

In this section, the relationship between sleep alterations and ADHD, OCD, eating disorders, anxiety, and adolescent depression in adolescence will be reviewed. As introduced previously, the incidence of all these psychiatric disorders nonlinearly increases from childhood to adolescence, and their prevalence in teens is higher relative to children and adults.

\section{Sleep and ADHD in children and adolescents}

ADHD is one of most common and socially important child psychiatric disorders. Onset is already observed in early childhood with a boy-to-girl ratio of 5:1. In schoolage children, prevalence rates are about $5 \%$ to $12 \%$, and, if untreated, ADHD crosses into adolescence and adulthood with more severe symptoms accompanied by substance abuse, antisocial behaviors, depression, and poor academic achievement. ${ }^{53,54,177,178}$ Along with its core symptoms, inattention, hyperactivity and impulsivity, altered sleep is a common feature in $\mathrm{ADHD}^{179-181}$ to the extent that sleep problems may be related to increase in ADHD symptoms during the day. ${ }^{182-186}$

Although sleep in ADHD is a subject of extensive research, the PSG data concerning alterations of sleep architecture in ADHD are largely inconsistent. Two metaanalyses did not provide conclusive views of whether ADHD is hallmarked by specific sleep changes. Rather, these meta-analyses showed that sleep in children with ADHD was characterized by high prevalence of OSA, PLMS, and 
RLS as well as by prolonged sleep onset, less actual time spent in sleep and worsened sleep efficiency, and frequent arousals. ${ }^{180,181}$ This inconsistency probably results from numerous confounders such as uncontrolled psychiatric and sleep-related comorbidities, loose diagnosis of ADHD without structured interviews, small sample sizes, inappropriate matching of groups for gender and age, and lack of medication control. ${ }^{179,181,187}$ Therefore, for the purpose of this review, we will present PSG findings from some studies where such confounders were minimized.

For example, Golan et al examined sleep architecture in 32 children and adolescents with ADHD (mean age: 12.4 years; 26 boys/8 girls) and 32 controls (mean age: 12 years; 21 boys/11 girls) and found more REM sleep in ADHD patients relative to controls. ${ }^{183}$ Of the ADHD patients, $15 \%$ had signs of sleep-disordered breathing occurring predominantly in REM sleep that was associated with their decreased vigilance on the next day as measured by multiple sleep latency test. ${ }^{183}$ Similarly, Kirov et al compared sleep pattern in 17 boys diagnosed with ADHD (mean age: 11.2 years) with 17 healthy boys (mean age: 11.2 years) and found an REM sleep increase in the ADHD patients. ${ }^{187}$ Later, the same authors also found an increased REM sleep in 18 children and adolescents with ADHD (mean age: 10.94 years; 17 boys/1 girl) compared with 18 controls (mean age: 11.58 years; 16 boys/ 2 girls). Importantly, the increased REM sleep in ADHD patients correlated positively with their core symptoms as assessed by Conners' Parents Rating Scale ${ }^{\circledR}$ and Child Behavior Checklist. ${ }^{185}$ By contrast, Gruber et al found less REM sleep in 15 prepubertal children (mean age: 8.93 years; 10 boys/5 girls) compared with 23 controls (mean age: 8.61 years; 13 boys/11 girls). ${ }^{188}$ More recent studies also show a relationship between sleep in children and adolescents with ADHD and their daily cognitive functioning. For example, Gruber et al showed that 1 hour of sleep deprivation in an ADHD cohort strongly reduced attention capabilities and increased ADHD symptoms from subclinical to clinical values. ${ }^{189}$ Another recent observation demonstrated that in children and adolescents with ADHD, REM sleep amount correlates positively with inattention scores and negatively with performance intelligence quotient. ${ }^{18}$

These findings demonstrate altered sleep architecture in children and adolescents with ADHD as well as decrements in arousal, cognitive, and psychological functioning in association with the sleep alterations. Thus, a bidirectional relationship between altered sleep in ADHD patients and their daily symptom expression and functioning may be suggested. Both REM sleep abnormalities and sleep disorders in ADHD may play roles in increasing and maintaining symptoms of ADHD. However, given the largely inconsistent sleep findings in ADHD, this issue needs further evaluation.

The DA agonist methylphenidate hydrochloride (MPH) is a drug of choice for treatment of ADHD. ${ }^{174,190}$ However, since it is evident that sleep alterations in ADHD also need to be treated, and because methylphenidate may cause adverse effects on sleep in ADHD through action on the mesopontine DA regulatory system, ${ }^{90,91}$ extended release formulations of methylphenidate appear promising. They are usually given in the mornings, and copy the daily expression of ADHD symptoms, presumably without effects on DA neuromodulation in the evenings, at habitual bedtimes, and during sleep. ${ }^{190,191}$ Finally, a recent review suggested that pharmacological treatment of ADHD with MPH had no short- or long-term effects on children's and adolescents' sleep. ${ }^{192}$

\section{Sleep in adolescents with OCD and eating disorders}

We discuss sleep changes in OCD and eating disorders together because they share common behavioral traits, obsession, and compulsion. OCD and eating disorders typically begin in adolescence or young adulthood, are seen in as many as 1 in 200 children and adolescents in relation to rapidly increased psychological vulnerability during this developmental period, and are frequently coexisting with other psychiatric conditions, including ADHD, TD, anxiety, and PTSD. ${ }^{33,52,180,193,194}$ Whereas much attention has been given to the relationship between sleep and many emotional and behavioral disturbances and psychiatric disorders including ADHD and depression in children and adolescents, less is known about sleep changes in adolescents with OCD and eating disorders. ${ }^{195-198}$

OCD is characterized by recurrent intense obsessions and/ or compulsions that cause severe discomfort and interfere with day-to-day functioning. In OCD, the obsessions and compulsions cause significant anxiety or distress, and they interfere with the child's normal routine, academic functioning, social activities, or relationships. ${ }^{177}$ Thus, it could be intuitively proposed that adolescents with OCD may be more likely than healthy peers to experience sleep problems for several reasons including heightened levels of OCD-related general anxiety and engagement in rituals before or during the bedtime routine that interfere with sleep onset. Although scarce, information about sleep alterations in adolescents with OCD confirms the above speculation. For example, a study of 18 pediatric OCD patients ( 9 adolescents with OCD and 9 matched healthy controls) reported that adolescents 
with OCD exhibited a number of sleep alterations including reduced sleep efficiency, increased sleep onset, and longer sleep latency to sleep stages. ${ }^{199}$ In another study examining OCD-related impairment across multiple domains, Piacentini et al reported that $66 \%$ of parents noted that OCD interfered with their child "getting ready for bed at night" and "sleeping at night". ${ }^{200}$ However, other studies in adolescent and adult OCD patients infer presence of disorder-specific changes in sleep. For example, shortened REM sleep latency and increased REM sleep as well as an overnight oversecretion of adrenocorticotropin (ACTH) and cortisol were observed in patients with $\mathrm{OCD}^{201-203}$ - these observations were discussed in relation to 5 -HT deficit in OCD. ${ }^{201}$ Thus, relationships between sleep architecture changes and OCD symptoms, particularly in adolescent patients, need further studies.

Eating disorders primarily affect adolescent girls and young adult females and are considered to be rare among males. These include AN, binge eating (BE) (also known as compulsive overeating), bulimia nervosa (BN), and sleeprelated eating disorder (SRED). AN is characterized by selfstarvation and excessive weight loss, including psychiatric symptoms such as intense fear of weight gain or being "fat" and feeling "fat" or overweight despite dramatic weight loss, and is behaviorally expressed by sore throat and painless swelling of the cheeks from vomiting. BE is primarily characterized by periods of uncontrolled, impulsive, or continuous eating beyond the point of feeling comfortably full. Behaviorally, it is characterized by eating large amounts of food when not physically hungry, rapid eating, feelings of disgust, depression, or guilt with overeating. Patients with $\mathrm{BE}$ usually do not purge afterward by vomiting or using laxatives. Diagnosis of $\mathrm{BN}$ is more difficult than $\mathrm{AN}$ and $\mathrm{BN}$; girls with $\mathrm{BN}$ try to remain at normal body weight or above while binge eating and purging. ${ }^{204}$ Patients with SRED generally arouse from sleep and eat roughly 2-3 hours after sleep onset. Eating episodes are characterized by rapid ingestion of food that the patient usually reports as "out of control" or compulsive in nature. ${ }^{204,198}$

Since general anxiety and engagement in rituals before or during the bedtime routine are common in eating disorders, ${ }^{198,205}$ the sleep alterations seen so far in female adolescents with AN, BE, BN, and SRED may be proposed to share common features with those observed in OCD adolescents. Indeed, a retrospective sleep evaluation by means of questionnaires in 400 young female adults with $\mathrm{AN}$ and $\mathrm{BN}$ revealed a generally disturbed sleep; ${ }^{196}$ this observation is consistent with previous PSG finding in females suffering from SRED. ${ }^{198}$ However, more precise sleep evaluation by means of PSG in female adolescents consistently found a reduction of EEG SWA $(0.5-4.5 \mathrm{~Hz})$, in which sleep alteration was associated with the neurobiological consequences of the malnourished state. ${ }^{206,207}$

Further concerning sleep changes in eating disorders, it should be emphasized that hypothalamic Hpcr/Orx neurons are involved in either sleep regulation or eating behavior, ${ }^{83}$ and a genetic study among females with eating disorders proposes the involvement of alterations in Hpcr/Orx system in these patients. ${ }^{208}$

The behavioral consequences from altered sleep in both OCD and eating disorders include daily somnolence and fatigue and worsened academic performance. ${ }^{196,198,209}$ Eating disorders accompanied by loss of weight or obesity in turn may produce sleep alterations. ${ }^{198,206,210}$

Pharmacotherapy for both OCD and eating disorders include 5-HT reuptake blockers and hypnotics and, particularly for the eating disorders, also DA agonists and codeine. ${ }^{201,211,212}$ However, benzodiazepine and nonbenzodiazepine hypnotics reduce SWS and REM sleep at the expense of lighter non-REM sleep stages, ${ }^{82}$ the effects of which are proposed to impair synaptic plasticity. ${ }^{213}$ Furthermore, psychostimulants are all shown to reduce REM sleep. ${ }^{214}$ Since non-REM and REM sleep regulatory mechanisms develop during adolescence and their normal overnight expression is important for growth and development, the pharmacotherapy in OCD and eating disorders should be supplemented by prevention of non-REM and REM sleep abnormalities. Prevention strategies may include psychological interviews, attendance at educational lectures, sleeping in presence of parents or peer healthy adolescents, and practicing physical exercises.

\section{Sleep in adolescents with anxiety disorders and depression}

The rate of anxiety disorders and adolescent depression rapidly increases in teens and is higher during this developmental period relative to childhood and adulthood. ${ }^{42}$ The rapid peaking of these psychiatric conditions in teens is associated with the behavioral changes that hallmark adolescence. ${ }^{32,40}$ Also, as mentioned earlier, it should be emphasized that anxiety disorders and adolescent depression may represent comorbid conditions in adolescent ADHD, TD, OCD, PTSD, and eating disorders.

Evidence from pediatric samples of patients with anxiety disorders suggests that sleep problems are prevalent and related to anxiety severity. Several studies examining the clinical presentation of pediatric generalized anxiety disorder 
have found rates of sleep disturbances to range from 42 to $66 \%{ }^{215-217}$ In a representative study of clinically anxious adolescents $(n=128)$ with generalized anxiety disorder, social phobia, and/or separation anxiety disorder, it was observed that $88 \%$ of youth complained of at least one sleep problem, with over $55 \%$ experiencing more than three sleep-related problems, including insomnia (66.6\%), nightmares $(54.5 \%)$, refusal to sleep alone (47.9\%), being overtired without good reason $(43.2 \%)$, refusal to sleep away from home $(40.9 \%)$, decreased sleep (36.9\%), and sleep walking/talking (22.7\%). Moreover, these sleep problems were positively related to measures of anxiety severity. ${ }^{218}$ Among nonclinical pediatric samples, Mindell and Barrett found a positive relationship between nightmares and trait anxiety, with more than three nightmares per week being related to parent proxy reports of clinically significant anxiety levels. ${ }^{219}$ Since it is unclear whether and how sleep problems in adolescents with anxiety disorders may affect their day-by-day well-being, this issue mandates focused investigations. Also, treatment of both anxiety disorders and related sleep problems in adolescence is so far overlooked because these problems are frequently included in the broad spectrum of behavioral changes hallmarking adolescence. However, high incidence of neuroticism associated with poor sleep and nightmares mandates increased attention because such behavioral and sleep problems may be preconditions for serious psychiatric disorders, including depression. ${ }^{138}$

The increased onset of depression at adolescence is a factor with highly increased risk of decrements in growth and development and further problems in adulthood. ${ }^{42}$ Early depressive episodes recur or persist into adult life along with ongoing psychosocial difficulties, including disruption in interpersonal relationships, low educational attainment, poor occupational functioning and unemployment, as well as elevated risk for suicidal behavior. ${ }^{220}$ Given the growing economic and psychosocial burden associated with juvenile depression, the identification of youth at highest risk for the illness will have great public health impact. ${ }^{221}$

Prospective epidemiological studies have demonstrated that sleep disturbances increase the risk for depressive disorders. ${ }^{222,223}$ Sleep PSG alterations found in adolescent depression were shown to be the same as in adults, and thus sleep measures appear an additional diagnostic tool. These sleep alterations include shortened latency to REM sleep and increased REM sleep amount, delayed sleep onset and insomnia, more time spent awake after sleep onset, and less SWS than in healthy subjects. ${ }^{138,224-226}$ Notably, sleep in depressive adolescents is shown to be additionally characterized by increased overnight occurrence of both $\mathrm{OSAs}^{227}$ and PLMS or RLS. ${ }^{131}$ A typical hypnogram of adolescent depressive patient is shown in Figure 1B. These sleep signatures of adolescent depression are accompanied by elevated HPA activity and increased cortisol levels. ${ }^{138,225}$ Further, depressive adolescents suffer from heavily increased incidence of nightmares compared with healthy peers, ${ }^{138,228,229}$ which is associated with the elevated REM sleep. ${ }^{230}$

The daily functioning of depressive patients is characterized by overall worsening of cognitive and academic abilities, as well as by emotional disregulation, which includes negative emotional tone and increased retrieval of emotional memories with negative affect. ${ }^{231,232}$ Also, patients with depression display a self-related memory consolidation instead of the normal consolidation of memories. ${ }^{231}$ Although it is not clear whether the worsened cognitive and psychological functioning in teens is disorder-associated or a result of the strongly altered sleep architecture, bidirectional relationships between these may be proposed.

Depression is a subject of extensive psychopharmacological treatment, including psychostimulants acting on 5-HT and NA reuptake mechanisms and benzodiazepine and nonbenzodiazepine hypnotics, in combination with psychotherapy. ${ }^{233}$ Given the complex role of sleep and its regulatory mechanisms for the normal physical, cognitive, and psychological functions during adolescence, timely management of sleep problems related to the behavioral and psychopathological conditions that rapidly undergo nonlinear changes in teens appears an important strategy for both minimizing the risk at depression and its prevention during this developmental period.

\section{Conclusion}

Adolescence is a critical growth and development period hallmarked by drastic and nonlinear hormonal, somatic, and behavioral changes, which are paralleled by dramatic maturational changes in sleep physiology, architecture, and regulatory mechanisms. Thus, sleep, in a complex association with many other processes, appears to play a critical role in normal functioning during adolescence.

There is a strong increase in many inappropriate behaviors, pathological somatic and psychological conditions, and psychiatric disorders in adolescence relative to childhood and adulthood. All these conditions are accompanied by more- or less-specific sleep alterations. Given that sleep and its specific architecture serves vitally important physiological, cognitive, and psychological processes, a bidirectional relationship between its maturational or pathological alterations and 
common somatic, cognitive, and psychiatric conditions in adolescence may be suggested to exist. Accordingly, the sleep alterations in these common adolescent pathological conditions may in turn worsen their symptom expression, and thus may lead to a number of additional somatic complications, poor academic achievement, and poor psychological wellbeing. Therefore, treatment of all these common adolescent conditions should also consider the alterations of sleep. This mandates an increased attention to both sleep changes in adolescence and common adolescent pathological conditions by parents, pediatricians, psychiatrists, and sleep researchers.

\section{Disclosure}

The authors report no conflicts of interest in this work.

\section{References}

1. Cirelli C, Tononi G. Is sleep essential? PLoS Biol. 2008;6:e216.

2. Siegel JM. Clues to the functions of mammalian sleep. Nature. 2005; 437:1264-1271.

3. Siegel JM. Sleep viewed as a state of adaptive inactivity. Nat Rev Neurosci. 2009;10:747-753.

4. Dinges DF, Rogers NL, Baynard MD. Chronic sleep deprivation. In: Kryger MH, Roth T, Dement WC, editors. Principles and Practice of Sleep Medicine. Philadelphia: Elsevier Saunders; 2005:67-76.

5. Hobson JA, Pace-Schott EF. The cognitive neuroscience of sleep: neuronal systems, consciousness and learning. Nat Rev Neurosci. 2002; 3:679-693.

6. Rechtschaffen A. Current perspectives on the function of sleep. Perspect Biol Med. 1998;41:359-390.

7. Tononi G, Cirelli C. Sleep function and synaptic homeostasis. Sleep Med Rev. 2006;10:49-62.

8. Diekelmann S, Born J. The memory function of sleep. Nat Rev Neurosci. 2010;11:114-126.

9. Maquet P. The role of sleep in learning and memory. Science. 2001; 294:1048-1052.

10. Krueger JM, Rector DM, Roy S, Van Dongen HP, Belenky G, Panksepp. Sleep as a fundamental property of neuronal assemblies. Nat Rev Neurosci. 2008;9:910-919.

11. Walker MP, Stickgold R. Sleep, memory, and plasticity. Annu Rev Psychol. 2006;57:139-166.

12. Brand S, Opwis K, Hatzinger M, Holsboer-Trachsler E. REM sleep is related to the transfer of implicit procedural knowledge following metacognitive learning. Somnologie. 2010;14:213-220.

13. Cai DJ, Mednick SA, Harrison EM, Kanady JC, Mednick SC. REM, not incubation, improves creativity by priming associative networks. Proc Natl Acad Sci US A. 2009;106:10130-10134.

14. Wagner U, Gais S, Haider H, et al. Sleep inspires insight. Nature. 2004; 427:352-355.

15. Yordanova J, Kolev V, Verleger R, Verleger R, Born J. Shifting from implicit to explicit knowledge: different roles of early- and late-night sleep. Learn Mem. 2008;15:508-515.

16. Bódizs R, Kis T, Lázár AS, et al. Prediction of general mental ability based on neural oscillation measures of sleep. J Sleep Res. 2005;14: 285-292.

17. Clemens Z, Fabó D, Halász P. Overnight verbal memory retention correlates with the number of sleep spindles. Neuroscience. 2005;132: 529-535.

18. Kirov R, Uebel H, Albrecht B, Banaschewski T, Rothenberger A. Two faces of REM sleep in normal and psychopathological development. Eur Psychiatry. 2011;26:422-423.
19. Mander BA, Santhanam S, Saletin JM, Walker MP. Wake deterioration and sleep restoration of human learning. Curr Biol. 2011;21: R183-R184.

20. Schabus M, Hödlmoser K, Gruber G, et al. Sleep spindle-related activity in the human EEG and its relation to general cognitive and learning abilities. Eur J Neurosci. 2006;23:1738-1746.

21. Schabus M, Hoedlmoser K, Pecherstorfer T, et al. Interindividual sleep spindle differences and their relation to learning-related enhancements. Brain Res. 2008;1191:127-135.

22. Nishida M, Pearsall J, Buckner RL, Walker MP. REM sleep, prefrontal theta, and the consolidation of human emotional memory. Cereb Cortex. 2009;19:1158-1166.

23. Wagner U, Gais S, Born J. Emotional memory formation is enhanced across sleep intervals with high amounts of rapid eye movement sleep. Learn Mem. 2001;8:112-119.

24. Walker MP. The role of sleep in cognition and emotion. Ann NY Acad Sci. 2009;1156:168-197.

25. Gujar N, McDonald SA, Nishida M, Walker MP. A role for rem sleep in recalibrating the sensitivity of the human brain to specific emotions. Cereb Cortex. 2011;21:115-123.

26. McNamara P, Andresen J, Clark J, Zborowski M, Duffy CA. Impact of attachment styles on dream recall and dream content: a test of the attachment hypothesis of REM sleep. J Sleep Res. 2001;10:117-127.

27. McNamara P, McLaren D, Smith D, Brown A, Stickgold R. A "Jekyll and Hyde" within: aggressive versus friendly interactions in REM and non-REM dreams. Psychol Sci. 2005;16:130-136.

28. van der Helm E, Gujar N, Walker MP. Sleep deprivation impairs the accurate recognition of human emotions. Sleep. 2010;33:335-342.

29. Mikoteit T, Brand S, Beck J, et al. Visually detected NREM Stage 2 sleep spindles in kindergarten children are associated with stress challenge and coping strategies. World J Biol Psychiatry. Epub ahead of print.

30. Gujar N, Yoo SS, Hu P, Walker MP. The unrested resting brain: sleep deprivation alters activity within the default-mode network. $J \operatorname{Cog} n$ Neurosci. 2010;22:1637-1648.

31. Ernst M, Pine DS, Hardin M. Triadic model of the neurobiology of motivated behavior in adolescence. Psychol Med. 2006;36:299-312.

32. Spear LP. The adolescent brain and age-related behavioral manifestations. Neurosci Biobehav Rev. 2000;24:417-463.

33. Pinyerd B, Zipf WB. Puberty-timing is everything! J Pediatr Nurs. 2005;20:75-82.

34. Romeo RD. Adolescence: a central event in shaping stress reactivity. Dev Psychobiol. 2010;52:244-253.

35. Casey BJ, Getz S, Galvan A. The adolescent brain. Dev Rev. 2008; 28:62-77.

36. Blakemore SJ. The developing social brain: implications for education. Neuron. 2010;65:744-747.

37. Burnett S, Sebastian C, Cohen Kadosh K, Blakemore SJ. The social brain in adolescence: evidence from functional magnetic resonance imaging and behavioural studies. Neurosci Biobehav Rev. Epub ahead of print.

38. White AM. Understanding adolescent brain development and its implications for the clinician. Adolesc Med State Art Rev. 2009;20:73-90.

39. Uhlhaas PJ, Roux F, Singer W, Haenschel C, Sireteanu R, Rodriguez E. The development of neural synchrony reflects late maturation and restructuring of functional networks in humans. Proc Natl Acad Sci US A. 2009;106:9866-9871.

40. Somerville LH, Jones RM, Casey BJ. A time of change: behavioral and neural correlates of adolescent sensitivity to appetitive and aversive environmental cues. Brain Cogn. 2010;72:124-133.

41. Compas BE, Orosan PG, Grant KE. Adolescent stress and coping: Implications for psychopathology during adolescence. J Adolesc. 1993; 16:331-349.

42. Kessler RC, Berglund P, Delmer O, Jin R, Merikangas KR, Walters EE. Lifetime prevalence and age-of-onset distributions of DSM-IV disorders in the National Comorbidity Survey Replication. Arch Gen Psychiatry. 2005;62:593-602. 
43. Paus T, Keshavan M, Giedd JN. Why do many psychiatric disorders emerge during adolescence? Nat Rev Neurosci. 2008;9:947-957.

44. Post RM, Leverich GS, Xing G, Weiss RB. Developmental vulnerabilities to the onset and course of bipolar disorder. Dev Psychopathol. 2001;13:581-598.

45. Beesdo K, Knappe S, Pine DS. Anxiety and anxiety disorders in children and adolescents: developmental issues and implications for DSM-V. Psychiatr Clin North Am. 2009;32:483-524.

46. Varchol L, Cooper H. Psychotherapy approaches for adolescents with eating disorders. Curr Opin Pediatr. 2009;21:457-464.

47. Vigod SN, Stewart DE. Emergent research in the cause of mental illness in women across the lifespan. Curr Opin Psychiatry. 2009; 22:396-400

48. Rajji TK, Ismail Z, Mulsant BH. Age at onset and cognition in schizophrenia: meta-analysis. Br J Psychiatry. 2009;195:286-293.

49. Spear LP. Heightened stress responsivity and emotional reactivity during pubertal maturation: Implications for psychopathology. Dev Psychopathol. 2009;21:87-97.

50. Angst J, Cui L, Swendsen J, Rothen S, et al. Major depressive disorder with subthreshold bipolarity in the National Comorbidity Survey Replication. Am J Psychiatry. 2010;167:1194-1201.

51. Chang KD. Course and impact of bipolar disorder in young patients. J Clin Psychiatry. 2010;71:e05.

52. Lock J, Fitzpatrick KK. Advances in psychotherapy for children and adolescents with eating disorders. Am J Psychother. 2009; 63:287-303

53. Bussing R, Mason DM, Bell L, Porter P, Garvan C. Adolescent outcomes of childhood attention-deficit/hyperactivity disorder in a diverse community sample. J Am Acad Child Adolesc Psychiatry. 2010;49:595-605.

54. Chronis-Tuscano A, Molina BS, Pelham WE, et al. Very early predictors of adolescent depression and suicide attempts in children with attention-deficit/hyperactivity disorder. Arch Gen Psychiatry. 2010; 67:1044-1051.

55. Leckman JF. Tourette's syndrome. Lancet. 2002;360:1577-1586.

56. Bloch MH, Leckman JF. Clinical course of Tourette syndrome. J Psychosom Res. 2009;67:497-501.

57. Laberge L, Petit D, Simard C, Vitaro F, Tremblay RE, Montplaisir J. Development of sleep patterns in early adolescence. J Sleep Res. 2001;10:59-67.

58. McLaughlin Crabtree V, Williams NA. Normal sleep in children and adolescents. Child Adolesc Psychiatr Clin N Am. 2009;18:799-811.

59. O’Brien EM, Mindell JA. Sleep and risk-taking behavior in adolescents. Behav Sleep Med. 2005;3:113-133.

60. Roffwarg HP, Muzio JN, Dement WC. Ontogenetic development of the human sleep-dream cycle. Science. 1966;152:604-619.

61. Tarokh L, Carskadon MA. Developmental changes in the human sleep EEG during early adolescence. Sleep. 2010;33:801-809.

62. Taylor DJ, Jenni OG, Acebo C, Carskadon MA. Sleep tendency during extended wakefulness: insights into adolescent sleep regulation and behavior. J Sleep Res. 2005;14:239-244.

63. Buchmann A, Ringli M, Kurth S, et al. EEG sleep slow-wave activity as a mirror of cortical maturation. Cereb Cortex. 2011;21: 607-615.

64. Feinberg I, Campbell IG. Sleep EEG changes during adolescence: an index of a fundamental brain reorganization. Brain Cogn. 2010; 72:56-65.

65. Feinberg I, de Bie E, Davis NM, Campbell IG. Topographic differences in the adolescent maturation of the slow wave EEG during NREM sleep. Sleep. 2011;34:325-333.

66. Kurth S, Jenni OG, Riedner BA, Tononi G, Carskadon MA, Huber R. Characteristics of sleep slow waves in children and adolescents. Sleep. 2010;33:475-480.

67. Rechtschaffen A, Kales A. A Manual of Standardized Terminology Techniques and Scoring System for Sleep Stages in Human Subjects. US Department of Health, Education and Welfare, Public Health Service. Washington DC US Government Printing Office; 1968.
68. Achermann P, Borbély AA. Low-frequency $(<1 \mathrm{~Hz})$ oscillations in the human sleep electroencephalogram. Neuroscience. 1997; 81:213-222

69. Steriade M, Nuñez A, Amzica F. A novel slow $(<1 \mathrm{~Hz})$ oscillation of neocortical neurons in vivo: depolarizing and hyperpolarizing components. J Neurosci. 1993;13:3252-3265.

70. Aserinsky E, Kleitman N. Regularly occurring periods of eye motility, and concomitant phenomena, during sleep. Science. 1953;118: 273-274.

71. Cantero JL, Atienza M, Stickgold R, Kahana MJ, Madsen JR, Kocsis B. Sleep-dependent theta oscillations in the human hippocampus and neocortex. J Neurosci. 2003;23:10897-10903.

72. Clemens Z, Weiss B, Szucs A, Eross L, Rásonyi G, Halász P. Phase coupling between rhythmic slow activity and gamma characterizes mesiotemporal rapid-eye-movement sleep in humans. Neuroscience. 2009; 163:388-396.

73. De Gennaro L, Ferrara M. Sleep spindles: an overview. Sleep Med Rev. 2003;7:423-440.

74. Broughton RJ. Polysomnography: principles and applications in sleep and arousal disorders. In: Niedermeyer E, Lopes da Silva F, editors. Electroencephalography: Basic Principles, Clinical Applications and Related Fields. Baltimore: Urban and Schwarzenberg; 1987:765-802.

75. Sinton CM, McCarley RW. Neuroanatomical and neurophysiological aspects of sleep: basic science and clinical relevance. Semin Clin Neuropsychiatry. 2000;5:6-19.

76. Borbély AA. A two process model of sleep regulation. Hum Neurobiol. 1982;1:195-204.

77. Borbély AA, Achermann P. Sleep homeostasis and models of sleep regulation. J Biol Rhythms. 1999;14:557-568.

78. Hobson JA. Sleep is of the brain, by the brain and for the brain. Nature. 2005;437:1254-1256.

79. Gottesmann C. Neurophysiological support of consciousness during waking and sleep. Prog Neurobiol. 1999;59:469-508.

80. Pace-Schott EF, Hobson JA. The neurobiology of sleep: genetics, cellular physiology and subcortical networks. Nat Rev Neurosci. 2002; 3:591-605.

81. Steiger A. Sleep and the hypothalamo-pituitary-adrenocortical system. Sleep Med Rev. 2002;6:125-138.

82. Steiger A. Neurochemical regulation of sleep. J Psychiatr Res. 2007; 41:537-552.

83. Mignot E, Taheri S, Nishino S. Sleeping with the hypothalamus: emerging therapeutic targets for sleep disorders. Nat Neurosci. 2002; 5 Suppl:1071-1075.

84. Higgings ES, George MS. The Neuroscience of Clinical Psychiatry. The Pathophysiology of Behavior and Mental Illness. Philadelphia: Lippincott Williams \& Wilkins; 2007.

85. McCormick DA, Bal T. Sleep and arousal: thalamocortical mechanisms. Annu Rev Neurosci. 1997;20:185-215.

86. Steriade M, Timofeev I. Neuronal plasticity in thalamocortical networks during sleep and waking oscillations. Neuron. 2003;37:563-576.

87. Hobson JA, McCarley RW, Wyzinski PW. Sleep cycle oscillation: reciprocal discharge by two brainstem neuronal groups. Science. 1975; 189:55-58.

88. Steriade M, Hobson J. Neuronal activity during the sleep-waking cycle. Prog Neurobiol. 1976;6:155-376.

89. Léna I, Parrot S, Deschaux O, et al. Variations in extracellular levels of dopamine, noradrenaline, glutamate, and aspartate across the sleepwake cycle in the medial prefrontal cortex and nucleus accumbens of freely moving rats. J Neurosci Res. 2005;81:891-899.

90. Monti JM, Monti D. The involvement of dopamine in the modulation of sleep and waking. Sleep Med Rev. 2007;11:113-133.

91. Rye DB. The two faces of Eve: dopamine's modulation of wakefulness and sleep. Neurology. 2004;63:S2-S7.

92. Kobayashi T, Good C, Biedermann J, Barnes C, Skinner RD, Garcia-Rill E. Developmental changes in pedunculopontine nucleus (PPN) neurons. J Neurophysiol. 2004;91:1470-1481. 
93. Kobayashi T, Good C, Mamiya K, Skinner RD, Garcia-Rill E. Development of REM sleep drive and clinical implications. J Appl Physiol. 2004;96:735-746.

94. Garcia-Rill E, Charlesworth A, Heister D, Ye M, Hayar A. The developmental decrease in REM sleep: the role of transmitters and electrical coupling. Sleep. 2008;31:673-690.

95. Hatzinger M, Hemmeter U, Brand S, Ising M, Holsboer-Trachsler E. Electroencephalo-graphic (EEG) sleep profiles in treatment course and long-term outcome of major depression: association with DEX/ CRH-test response. J Psychiatr Res. 2004;38:453-465.

96. Carskadon MA, Acebo C. Regulation of sleepiness in adolescents: update, insights, and speculation. Sleep. 2002;25:606-614.

97. Carskadon MA, Acebo C, Jenni OG. Regulation of adolescent sleep: implications for behavior. In: Dahl RE, Spear LP, editors. Adolescent Brain Development: and Opportunities. NY: New York Academy of Science; 2004:274-291.

98. Harvey AG. A cognitive model of insomnia. Behav Res Ther. 2002; 40(8):869-893

99. Riemann D, Spiegelhalder K, Feige B, et al. The hyperarousal model of insomnia: a review of the concept and its evidence. Sleep Med Rev. 2010;14:19-31.

100. Ribeiro S, Mello CV, Velho T, Gardner TJ, Jarvis ED, Pavlides C. Induction of hippocampal long-term potentiation during waking leads to increased extrahippocampal zif-268 expression during ensuing rapid-eye-movement sleep. J Neurosci. 2002;22:10914-10923.

101. Ribeiro S, Nicolelis MA. Reverberation, storage, and postsynaptic propagation of memories during sleep. Learn Mem. 2004;11: 686-696.

102. Marshall L, Helgadóttir H, Mölle M, Born J. Boosting slow oscillations during sleep potentiates memory. Nature. 2006;444:610-613.

103. Marshall L, Kirov R, Brade J, Mölle M, Born J. Transcranial electrical currents to probe EEG brain rhythms and memory consolidation during sleep in humans. PLoS One. 2011;6(2):e16905.

104. Wilhelm I, Diekelmann S, Molzow I, Ayoub A, Mölle M, Born J Sleep selectively enhances memory expected to be of future relevance. J Neurosci. 2011;31:1563-1569.

105. Diekelmann S, Büchel C, Born J, Rasch B. Labile or stable: opposing consequences for memory when reactivated during waking and sleep. Nat Neurosci. 2011;14:381-386

106. Rasch B, Büchel C, Gais S, Born J. Odor cues during slow-wave sleep prompt declarative memory consolidation. Science. 2007;315: 1426-1429.

107. Peigneux P, Laureys S, Fuchs S, et al. Are spatial memories strengthened in the human hippocampus during slow wave sleep? Neuron. 2004; 44:535-545.

108. Marshall L, Born J. The contribution of sleep to hippocampus-dependent memory consolidation. Trends Cogn Sci. 2007;11:442-450.

109. Rasch B, Born J. Maintaining memories by reactivation. Curr Opin Neurobiol. 2007;17:698-703.

110. Yordanova J, Kolev V, Wagner U, Born J, Verleger R. Increased alpha $(8-12 \mathrm{~Hz})$ activity during slow-wave sleep as a marker for the transition from implicit knowledge to explicit insight. J Cogn Neurosci. In press.

111. Fogel SM, Smith CT. Learning-dependent changes in sleep spindles and Stage 2 sleep. J Sleep Res. 2006;15:250-255.

112. Hobson JA, Pace-Schott EF, Stickgold R. Dreaming and the brain: toward a cognitive neuroscience of conscious states. Behav Brain Sci. 2000;23:793-842; discussion 904-1121.

113. Stickgold R, Hobson JA, Fosse R, Fosse M. Sleep, learning, and dreams: off-line memory reprocessing. Science. 2001;294:1052-1057.

114. Stickgold R, Scott L, Rittenhouse C, Hobson JA. Sleep-induced changes in associative memory. J Cogn Neurosci. 1999;11:182-193.

115. Walker MP, Liston C, Hobson JA, Stickgold R. Cognitive flexibility across the sleep-wake cycle: REM-sleep enhancement of anagram problem solving. Brain Res Cogn Brain Res. 2002;14:317-324.

116. Fischer S, Hallschmid M, Elsner AL, Born J. Sleep forms memory for finger skills. Proc Natl Acad Sci U S A. 2002;99:11987-11991.
117. Karni A, Tanne D, Rubenstein BS, Askenasy JJ, Sagi D. Dependence on REM sleep of overnight improvement of a perceptual skill. Science. 1994;265:679-682.

118. Maquet P, Laureys S, Peigneux P, et al. Experience-dependent changes in cerebral activation during human REM sleep. Nat Neurosci. 2000; 3:831-836

119. Plihal W, Born J. Effects of early and late nocturnal sleep on priming and spatial memory. Psychophysiology. 1999;36:571-582.

120. Smith CT, Nixon MR, Nader RS. Posttraining increases in REM sleep intensity implicate REM sleep in memory processing and provide a biological marker of learning potential. Learn Mem. 2004; 11:714-719.

121. Rasch B, Pommer J, Diekelmann S, Born J. Pharmacological REM sleep suppression paradoxically improves rather than impairs skill memory. Nat Neurosci. 2009;12:396-397.

122. Rasch B, Gais S, Born J. Impaired off-line consolidation of motor memories after combined blockade of cholinergic receptors during REM sleep-rich sleep. Neuropsychopharmacology. 2009;34: 1843-1853.

123. Walker MP, Stickgold R. Overnight alchemy: sleep-dependent memory evolution. Nat Rev Neurosci. 2010;11:218; author reply 218.

124. Cartwright R, Agargun MY, Kirkby J, Friedman JK. Relation of dreams to waking concerns. Psychiatry Res. 2006;141:261-270.

125. Wagner U, Hallschmid M, Rasch B, Born J. Brief sleep after learning keeps emotional memories alive for years. Biol Psychiatry. 2006;60:788-790.

126. Walker MP, van der Helm E. Overnight therapy? The role of sleep in emotional brain processing. Psychol Bull. 2009;135:731-748.

127. Walker MP, Harvey AG. Obligate symbiosis: sleep and affect. Sleep Med Rev. 2010;14:215-217.

128. Cain N, Gradisar M. Electronic media use and sleep in school-aged children and adolescents: A review. Sleep Med. 2010;11:735-742.

129. Gromov I, Gromov D. Sleep and substance use and abuse in adolescents. Child Adolesc Psychiatr Clin N Am. 2009;18:929-946.

130. Gruber R, Wiebe ST, Wells SA, Cassoff J, Monson E. Sleep and academic success: mechanisms, empirical evidence, and interventional strategies. Adolesc Med State Art Rev. 2010;21:522-541.

131. Lofthouse N, Gilchrist R, Splaingard M. Mood-related sleep problems in children and adolescents. Child Adolesc Psychiatr Clin NAm. 2009; 18:893-916.

132. O'Brien LM. The neurocognitive effects of sleep disruption in children and adolescents. Child Adolesc Psychiatr Clin N Am. 2009;18: 813-823.

133. Owens JA. Etiologies and evaluation of sleep disturbances in adolescence. Adolesc Med State Art Rev. 2010;21:430-445.

134. Jan JE, Reiter RJ, Bax MC, Ribary U, Freeman RD, Wasdell MB. Long-term sleep disturbances in children: a cause of neuronal loss. Eur J Paediatr Neurol. 2010;14:380-390.

135. Hayes D Jr. Impact of inadequate sleep on unintentional injuries in adolescents. Adolesc Med State Art Rev. 2010;21:491-507.

136. Shibley HL, Malcolm RJ, Veatch LM. Adolescents with insomnia and substance abuse: consequences and comorbidities. J Psychiatr Pract. 2008; $14: 146-153$.

137. Kirov R, Brand S. Nightmares as predictors of psychiatric disorders in adolescence. Curr Trends Neurol. In press.

138. Rao U, Hammen CL, Poland RE. Risk markers for depression in adolescents: sleep and HPA measures. Neuropsychopharmacology. 2009;34:1936-1945.

139. Kaneita Y, Yokoyama E, Harano S, et al. Associations between sleep disturbance and mental health status: a longitudinal study of Japanese junior high school students. Sleep Med. 2009;10:780-786.

140. Brand S, Beck J, Gerber M, Hatzinger M, Holsboer-Trachsler E. Evidence of favorable sleep-EEG patterns in adolescent male vigorous football players compared to controls. World J Biol Psychiatry. 2010 11(2 Pt 2):465-475.

141. Mossey PA, Little J, Munger RG, Dixon MJ, Shaw WC. Cleft lip and palate. Lancet. 2009;374:1773-1785. 
142. Eurocat. European Surveillance of Congenital Anomalies. http://www. eurocat-network.eu/. Accessed June 6, 2010.

143. Brand S, Blechschmidt A, Sader R, et al. Psychosocial functioning and sleep patterns in children and adolescents with cleft lip and palate (CLP) compared to healthy controls. Cleft Palate Craniofac J. 2009; 46:125-135.

144. Brand S, Müller AA. Quality of life among children, adolescents and adults with orofacial clefts. In: Stone JH, Blouin M, editors. International Encyclopedia of Rehabilitation. 2011. http://cirrie.buffalo.edu/ encyclopedia/en/article/294/.

145. Maclean JE, Waters K, Fitzsimons D, Hayward P, Fitzgerald DA. Screening for obstructive sleep apnea in preschool children with cleft palate. Cleft Palate Craniofac J. 2009;46(2):117-123.

146. MacLean JE, Fitzsimons D, Hayward P, Waters KA, Fitzgerald DA. The identification of children with cleft palate and sleep disordered breathing using a referral system. Pediatr Pulmonol. 2008;43:245-250.

147. Muntz H, Wilson M, Park A, Smith M, Grimmer JF. Sleep disordered breathing and obstructive sleep apnea in the cleft population. Laryngoscope. 2008;118:348-353.

148. Coebergh JW, Reedijk AM, de Vries E, et al. Leukaemia incidence and survival in children and adolescents in Europe during 1978-1997. Report from the Automated Childhood Cancer Information System project. Eur J Cancer. 2006;42:2019-2036.

149. Walker AJ, Johnson KP, Miaskowski C, Lee KA, Gedaly-Duff V. Sleep quality and sleep hygiene behaviors of adolescents during chemotherapy. J Clin Sleep Med. 2010;15:439-444.

150. Zupanec S, Jones H, Stremler R. Sleep habits and fatigue of children receiving maintenance chemotherapy for ALL and their parents. J Pediatr Oncol Nurs. 2010;27:217-228.

151. Rosen G, Brand SR. Sleep in children with cancer: case review of 70 children evaluated in a comprehensive pediatric sleep center. Support Care Cancer. Epub ahead of print.

152. Urschitz MS, Brockmann PE, Schlaud M, Poets CF. Population prevalence of obstructive sleep apnoea in a community of German third graders. Eur Respir J. 2010;36:556-568.

153. Tran KD, Nguyen CD, Weedon J, Goldstein NA. Child behavior and quality of life in pediatric obstructive sleep apnea. Arch Otolaryngol Head Neck Surg. 2005;131:52-57.

154. Ali NJ, Pitson D, Stradling JR. Sleep disordered breathing: effects of adenotonsillectomy on behaviour and psychological functioning. Eur J Pediatr. 1996;155:56-62.

155. Beebe DW. Neurobehavioral morbidity associated with disordered breathing during sleep in children: a comprehensive review. Sleep. 2006;29:1115-1134.

156. Pagel JF, Snyder S, Dawson D. Obstructive sleep apnea in sleepy pediatric psychiatry clinic patients: polysomnographic and clinical correlates. Sleep Breath. 2004;8:125-131.

157. Huang QR, Qin Z, Zhang S, Chow CM. Clinical patterns of obstructive sleep apnea and its comorbid conditions: a data mining approach. J Clin Sleep Med. 2008;4:543-550.

158. Kheirandish-Gozal L, De Jong MR, Spruyt K, Chamuleau SA, Gozal D. Obstructive sleep apnoea is associated with impaired pictorial memory task acquisition and retention in children. Eur Respir J. 2010;36:149-164.

159. Spicuzza L, Leonardi S, La Rosa M. Pediatric sleep apnea: early onset of the 'syndrome'? Sleep Med Rev. 2009;13:111-122.

160. Bhattacharjee R, Kim J, Kheirandish-Gozal L, Gozal D. Obesity and obstructive sleep apnea syndrome in children: a tale of inflammatory cascades. Pediatr Pulmonol. 2011;46:313-323.

161. Mitchell RB. Adenotonsillectomy for obstructive sleep apnea in children: outcome evaluated by pre- and postoperative polysomnography. Laryngoscope. 2007;117:1844-1854.

162. O'Donnell AR, Bjornson CL, Bohn SG, Kirk VG. Compliance rates in children using noninvasive continuous positive airway pressure. Sleep. 2006;29:651-658.

163. Beebe DW, Byars KC. Adolescents with obstructive sleep apnea adhere poorly to positive airway pressure (PAP), but PAP users show improved attention and school performance. PLoS One. 2011;6:e16924.
164. Blunden S, Lushington K, Lorenzen B, Martin J, Kennedy D. Neuropsychological and psychosocial function in children with a history of snoring or behavioral sleep problems. J Pediatr. 2005; 146(6):780-786.

165. Allen RP, Picchietti D, Hening WA, et al. Restless legs syndrome: diagnostic criteria, special considerations, and epidemiology. A report from the restless legs syndrome diagnosis and epidemiology workshop at the National Institutes of Health. Sleep Med. 2003;4:101-119.

166. Hening WA, Allen RP, Earley CJ, Picchietti DL, Silber MH, Restless Legs Syndrome Task Force of the Standards of Practice Committee of the American Academy of Sleep Medicine. An update on the dopaminergic treatment of restless legs syndrome and periodic limb movement disorder. Sleep. 2004;27:560-583.

167. Konofal E, Lecendreux M, Arnulf I, Mouren MC. Iron deficiency in children with attention-deficit/hyperactivity disorder. Arch Pediatr Adolesc Med. 2004;58:1113-1115.

168. Konofal E, Cortese S, Marchand M, Mouren MC, Arnulf I, Lecendreux M. Impact of restless legs syndrome and iron deficiency on attention-deficit/hyperactivity disorder in children. Sleep Med. 2007;(7-8):711-715.

169. Michaud M, Soucy JP, Chabli A, Lavigne G, Montplaisir J. SPECT imaging of striatal pre- and postsynaptic dopaminergic status in restless legs syndrome with periodic leg movements in sleep. $J$ Neurol. 2002;249:164-170.

170. Picchietti MA, Picchietti DL. Advances in pediatric restless legs syndrome: Iron, genetics, diagnosis and treatment. Sleep Med. 2010; 11:643-651.

171. Stiasny K, Oertel WH, Trenkwalder C. Clinical symptomatology and treatment of restless legs syndrome and periodic limb movement disorder. Sleep Med Rev. 2002;6:253-265.

172. Chervin RD, Archbold KH, Dillon JE, et al. Associations between symptoms of inattention, hyperactivity, restless legs, and periodic leg movements. Sleep. 2002;25:213-218.

173. Picchietti DL, England SJ, Walters AS, Willis K, Verrico T. Periodic limb movement disorder and restless legs syndrome in children with attention-deficit hyperactivity disorder. J Child Neurol. 1998; 13:588-594.

174. Biederman J, Faraone SV. Attention-deficit hyperactivity disorder. Lancet. 2005;366:237-248.

175. Cortese S, Lecendreux M, Bernardina BD, Mouren MC, Sbarbati A, Konofal E. Attention-deficit/hyperactivity disorder, Tourette's syndrome, and restless legs syndrome: the iron hypothesis. Med Hypotheses. 2008;70:1128-1132.

176. Yilmaz K, Kilincaslan A, Aydin N, Kor D. Prevalence and correlates of restless legs syndrome in adolescents. Dev Med Child Neurol. 2011; 53(1):40-47.

177. American Psychiatric Association. Diagnostic and Statistical Manual of Mental Disorders. 4th ed. Washington DC: American Psychiatric Association; 1994.

178. National Institutes of Health Consensus Development Conference Statement: diagnosis and treatment of attention-deficit/hyperactivity disorder (ADHD). J Am Acad Child Adolesc Psychiatry. 2000;39:182-193.

179. Cohen-Zion M, Ancoli-Israel S. Sleep in children with attention-deficit hyperactivity disorder (ADHD): a review of naturalistic and stimulant intervention studies. Sleep Med Rev. 2004;8:379-402.

180. Cortese S, Faraone SV, Konofal E, Lecendreux M. Sleep in children with attention-deficit/hyperactivity disorder: meta-analysis of subjective and objective studies. JAm Acad Child Adolesc Psychiatry. 2009; 48:894-908.

181. Sadeh A, Pergamin L, Bar-Haim Y. Sleep in children with attentiondeficit hyperactivity disorder: a meta-analysis of polysomnographic studies. Sleep Med Rev. 2006;10:381-398.

182. Brown TE, McMullen WJ Jr. Attention deficit disorders and sleep/ arousal disturbance. Ann NY Acad Sci. 2001;931:271-286.

183. Golan N, Shahar E, Ravid S, Pillar G. Sleep disorders and daytime sleepiness in children with attention-deficit/hyperactive disorder. Sleep. 2004;27:261-266. 
184. Konofal E, Lecendreux M, Cortese S. Sleep and ADHD. Sleep Med. 2010;11:652-658.

185. Kirov R, Kinkelbur J, Banaschewski T, Rothenberger A. Sleep patterns in children with attention-deficit/hyperactivity disorder, tic disorder, and comorbidity. J Child Psychol Psychiatry. 2007;48:561-570.

186. Rothenberger A, Kirov R. Changes in sleep-wake behavior may be more than just an epiphenomenon of ADHD. Behav Brain Sci. 2005; 28:439.

187. Kirov R, Kinkelbur J, Heipke S, et al. Is there a specific polysomnographic sleep pattern in children with attention deficit/hyperactivity disorder? J Sleep Res. 2004;13:87-93.

188. Gruber R, Xi T, Frenette S, Robert M, Vannasinh P, Carrier J. Sleep disturbances in prepubertal children with attention deficit hyperactivity disorder: a home polysomnography study. Sleep. 2009;32:343-350.

189. Gruber R, Wiebe S, Montecalvo L, Brunetti B, Amsel R, Carrier J. Impact of sleep restriction on neurobehavioral functioning of children with attention deficit hyperactivity disorder. Sleep. 2011;34: 315-323.

190. MTA Cooperative Group. Moderators and mediators of treatment response for children with attention-deficit/hyperactivity disorder: The Multimodal Treatment Study of children with attention-deficit hyperactivity disorder. Arc Gen Psychiatry. 1999;56:1088-1096.

191. Swanson J, Gupta S, Lam A, et al. Development of a new once-a-day formulation of methylphenidate for the treatment of attention-deficit/ hyperactivity disorder: proof-of-concept and proof-of-product studies. Arch Gen Psychiatry. 2003;60:204-211.

192. Huang YS, Tsai MH, Guilleminault C. Pharmacological treatment of ADHD and short and long term effects on sleep. Curr Pharm Design. Epub ahead of print.

193. Fisher M, Golden NH, Katzman DK, et al. Eating disorders in adolescents: a background paper. J Adolesc Health. 1995;16:420-437.

194. Young J. Common comorbidities seen in adolescents with attentiondeficit/hyperactivity disorder. Adolesc Med State Art Rev. 2008; 19:216-228.

195. Ivanenko A, Crabtree VM, Gozal D. Sleep in children with psychiatric disorders. Pediatr Clin North Am. 2004;51:51-68.

196. Kim KR, Jung YC, Shin MY, Namkoong K, Kim JK, Lee JH. Sleep disturbance in women with eating disorder: prevalence and clinical characteristics. Psychiatry Res. 2010;176:88-90.

197. Storch EA, Murphy TK, Lack CW, Geffken GR, Jacob ML, Goodman WK. Sleep-related problems in pediatric obsessive-compulsive disorder. J Anxiety Disord. 2008;22:877-885.

198. Winkelman JW, Johnson EA, Richards LM. Sleep-related Eating Disorder. In: Montagna P, Chokroverty S, editors. Sleep Disorders, Part 1. Handbook of Clinical Neurology Series. 2011;98: 385-577.

199. Rapoport J, Elkins R, Langer DH, et al. Childhood obsessive compulsive disorder. Am J Psychiatry. 1981;138:1545-1554.

200. Piacentini J, Bergman RL, Keller M, McCracken J. Functional impairment in children and adolescents with obsessive-compulsive disorder. J Child Adolesc Psychopharmacology. 2003;13:S61-S69.

201. Voderholzer U, Riemann D, Huwig-Poppe C, et al. Sleep in obsessive compulsive disorder: polysomnographic studies under baseline conditions and after experimentally induced serotonin deficiency. Eur Arch Psychiatry Clin Neurosci. 2007;257:173-182.

202. Kluge M, Schüssler P, Dresler M, Yassouridis A, Steiger A. Sleep onset REM periods in obsessive compulsive disorder. Psychiatry Res. 2007;152:29-35.

203. Kluge M, Schüssler P, Künzel HE, Dresler M, Yassouridis A, Steiger A. Increased nocturnal secretion of ACTH and cortisol in obsessive compulsive disorder. J Psychiatr Res. 2007;41(11):928-933.

204. American Psychiatric Association. Diagnostic and Statistical Manual of Mental Disorders. (DSM-IV-TR). Washington DC: American Psychiatric Association; 2000.

205. Sassaroli S, Ruggiero GM, Vinai P, et al. Daily and nightly anxiety among patients affected by night eating syndrome and binge eating disorder. Eat Disord. 2009;17:140-145.
206. Nobili L, Baglietto MG, De Carli F, et al. A quantified analysis of sleep electroencephalography in anorectic adolescents. Biol Psychiatry. 1999;45:771-775.

207. Nobili L, Baglietto MG, Beelke M, et al. Impairment of the production of delta sleep in anorectic adolescents. Sleep. 2004;27: 1539- 1553.

208. Janas-Kozik M, Stachowicz M, Mazurek U, et al. Preliminary study of the expression of genes connected with the orexigenic and anorexigenic system using microarray technique in anorexia nervosa. Neuropsychobiology. 2008;57:116-120.

209. Mukhopadhyay S, Fineberg NA, Drummond LM, et al. Delayed sleep phase in severe obsessive-compulsive disorder: a systematic case-report survey. CNS Spectr. 2008;13:406-413.

210. Schenck CH, Mahowald MW. Review of nocturnal sleep-related eating disorders. Int J Eat Disord. 1994;15:343-356.

211. Dennis K, Le Grange D, Bremer J. Olanzapine use in adolescent anorexia nervosa. Eat Weight Disord. 2006;11:e53-e56.

212. Powers PS, Bruty H. Pharmacotherapy for eating disorders and obesity. Child Adolesc Psychiatr Clin N Am. 2009;18:175-187.

213. Seibt J, Aton SJ, Jha SK, Coleman T, Dumoulin MC, Frank MG. The non-benzodiazepine hypnotic zolpidem impairs sleep-dependent cortical plasticity. Sleep. 2008;31:1381-1391.

214. Nielsen TA. A review of mentation in REM and NREM sleep: "covert" REM sleep as a possible reconciliation of two opposing models. Behav Brain Sci. 2000;23:851-866; discussion 904-1121.

215. Kendall PC, Pimentel SS. On the physiological symptom constellation in youth with Generalized Anxiety Disorder (GAD). JAnxiety Disord. 2003;17:211-221.

216. Masi G, Perugi G, Toni C, et al. Obsessive-compulsive bipolar comorbidity: Focus on children and adolescents. J Affect Disord. 2004; 78: 175-183.

217. Pina AA, Silverman WK, Fuentes RM, Kurtines WM, Weems CF. Exposure-based cognitive-behavioral treatment for phobic and anxiety disorders: treatment effects and maintenance for Hispanic/Latino relative to European-American youths. $J$ Am Acad Child Adolesc Psychiatry. 2003;42:1179-1187.

218. Alfano CA, Ginsburg GS, Kingery JN. Sleep-related problems among children and adolescents with anxiety disorders. J Am Acad Child Adolesc Psychiatry. 2007;46:224-232.

219. Mindell JA, Barret KM. Nightmares and anxiety in elementary-aged children: Is there a relationship? Child Care Health Dev. 2002; 28:317-322.

220. Rao U, Chen LA. Characteristics, correlates, and outcomes of childhood and adolescent depressive disorders. Dialogues Clin Neurosci. 2009;11:45-62.

221. Rao U. Development and natural history of pediatric depression: treatment implications. Clin Neuropsychiatry. 2006;3:194-204.

222. Breslau N, Roth T, Rosenthal L, Andreski P. Sleep disturbance and psychiatric disorders: a longitudinal epidemiological study of young adults. Biol Psychiatry. 1996;39:411-418.

223. Buysse DJ, Angst J, Gama A, Ajdacic V, Eich D, Rössler W. Prevalence, course and comorbidity of insomnia and depression in young adults. Sleep. 2008;31:473-480.

224. Kutcher S, Williamson P, Marton P, Szalai J. REM latency in endogenously depressed adolescents. Br J Psychiatry. 1992;161: 399-402.

225. Rao U, Poland RE. Electroencephalographic sleep and hypothalamic-pituitary-adrenal changes from episode to recovery in depressed adolescents. J Child Adolesc Psychopharmacol. 2008;18: 607-613.

226. Turner J, Drummond LM, Mukhopadhyay S, et al. A prospective study of delayed sleep phase syndrome in patients with severe resistant obsessive-compulsive disorder. World Psychiatry. 2007;6:108-111.

227. Nasr S, Wendt B, Kora S. Increased incidence of sleep apnea in psychiatric outpatients. Ann Clin Psychiatry. 2010;22:29-32.

228. Agargün MY, Cilli AS, Kara H, Tarhan N, Kincir F, Oz H. Repetitive frightening dreams and suicidal behavior in patients with major depression. Compr Psychiatry. 1998;39:198-202. 
229. Agargun MY, Besiroglu L, Cilli AS, et al. Nightmares, suicide attempts, and melancholic features in patients with unipolar major depression. $J$ Affect Disord. 2007;98:267-270.

230. McNamara P, Auerbach S, Johnson P, Harris E, Doros G. Impact of REM sleep on distortions of self-concept, mood and memory in depressed/anxious participants. J Affect Disord. 2010;122: 198-207.
231. Hornung OP, Regen F, Danker-Hopfe H, Heuser I, Anghelescu I. Sleep-related memory consolidation in depression: an emerging field of research. Depress Anxiety. 2008;25:E163-E165.

232. Gotlib IH, Joormann J. Cognition and depression: current status and future directions. Annu Rev Clin Psychol. 2010;27:285-312.

233. Campo JV, Bridge JA. Treatment of youth depression. Am J Psychiatry. 2009;166:958-960.

\section{Publish your work in this journal}

The International Journal of General Medicine is an international, peer-reviewed open-access journal that focuses on general and internal medicine, pathogenesis, epidemiology, diagnosis, monitoring and treatment protocols. The journal is characterized by the rapid reporting of reviews, original research and clinical studies across all disease areas.
A key focus is the elucidation of disease processes and management protocols resulting in improved outcomes for the patient. The manuscript management system is completely online and includes a very quick and fair peer-review system. Visit http://www.dovepress.com/ testimonials.php to read real quotes from published authors.

Submit your manuscript here: http://www.dovepress.com/international-journal-of-general-medicine-journal 